\title{
Atorvastatin and trans-caryophyllene for the prevention of leukopenia in an experimental chemotherapy model in Wistar rats
}

\author{
MARIA INES DA CRUZ CAMPOS ${ }^{1}$, WELLINGTON DORIGHETO ANDRADE VIEIRA ${ }^{2}$, CELSO NEIVA CAMPOS ${ }^{3}$, \\ FERNANDO MONTEIRO AARESTRUP ${ }^{4}$ and BEATRIZ JULIÃO VIEIRA AARESTRUP ${ }^{4,5}$ \\ ${ }^{1}$ Postgraduate Program in Health; ${ }^{2}$ School of Dentistry, Faculty of Medical and Health Sciences (PPGS); \\ ${ }^{3}$ Department of Dental Clinic, Dental Scool; ${ }^{4}$ Laboratory of Immunopathology and Clinical Immunology; \\ ${ }^{5}$ Department of Morphology, Institute of Biological Sciences, Federal University of Juiz de Fora, Juiz de Fora, MG, Brazil
}

Received November 16, 2014; Accepted January 14, 2015

DOI: $10.3892 / \mathrm{mco} .2015 .544$

\begin{abstract}
Malignant neoplasia represents the second cause of disease-related mortality and, among all patients diagnosed with cancer, $70 \%$ will receive chemotherapy during the course of treatment. As a consequence, an increasing number of researchers have focused their attention on the search for more specific anticancer therapies associated with fewer side effects. Leukopenia is an important adverse effect associated with chemotherapy. Secondary infection is very common among leukopenic patients, directly affecting the continuity of the chemotherapeutic treatment and leading to possible complications in tumor immune defense. Atorvastatin, a type of statin, is a known agent used to control hypercholesterolemia. Trans-caryophyllene, isolated from a resinous oil extracted from the Copaiba tree, possesses anti-inflammatory and analgesic properties. The aim of the present study was to evaluate, through a complete leukocyte count, the systemic immunomodulation potential of pentoxifylline (PTX), atorvastatin and trans-caryophyllene, as well as the possible prophylactic role of these drugs against secondary leukopenia, in an experimental chemotherapy model induced by 5 -fluorouracil (5-FU) in Wistar rats. A total of 32 male Wistar rats were used, 24 of which were submitted to treatment with atorvastatin, PTX and trans-caryophyllene prior to the administration of chemotherapy. The Shapiro-Wilk test was used to verify normality and the Kruskal-Wallis test was used for negative data in the normality test. Among the drugs selected, atorvastatin exhibited the best preventive potential in regards to leukopenia secondary to experimental chemotherapy induced by $5-\mathrm{FU}$, in
\end{abstract}

Correspondence to: $\operatorname{Dr}$ Beatriz Julião Vieira Aarestrup, Department of Morphology, Institute of Biological Sciences, Federal University of Juiz de Fora, José Lourenço Kelmer street, University Campus, São Pedro, 36036-900 Juiz de Fora, MG, Brazil

E-mail: beatrizaarestrup@gmail.com

Key words: chemotherapy, leukopenia, oral mucositis, atorvastatin, trans-caryophyllene comparison to the group receiving saline solution, while PTX amplified such alterations in the leukograms of the animals in this trial.

\section{Introduction}

Malignant neoplasia represents the second cause of disease-related mortality worldwide and, among all patients diagnosed with cancer, $70 \%$ will receive chemotherapy during the course of treatment (1-3). As a consequence, an increasing number of researchers in several countries have focused their attention on the search for more specific anticancer therapies associated with fewer side effects $(3,4)$.

Among the choices of chemotherapy, methotrexate and 5-fluorouracil (5-FU) are considered as safe drugs, with effective antimitotic activity, although they act on malignant tumor cells as well as normal cells exhibiting unstable mitotic activity, such as several glandular epithelial and lining cells, as well as hematopoietic cells originating in the bone marrow (4).

Leukopenia is a significant adverse effect observed during chemotherapy. Secondary infection is very commom among leukopenic patients, directly affecting the continuity of the chemotherapeutic treatment and leading to possible complications in tumor immune defense (5). Several authors consider leukopenia secondary to chemotherapy as one of the main causes of the anticancer treatment discontinuation, emphasizing the need to monitor the immune competence of the patient through a complete blood count (CBC) during the course of treatment $(3,4,6,7)$.

Chemotherapy is the standard treatment for cancer and leukopenia is the most common side effect, which is directly associated with patient survival (8). Consequently, the oncological experimental research has allowed the establishment of more effective therapeutic protocols, as well as the modulation of biological reactions and its effects on improved clinical results, combined with fewer side effects $(9,10)$.

Among all anticancer drugs, 5-FU has been the primary drug of choice in experimental chemotherapy models, due to its wide clinical application and low cost $(1,10,11)$.

In an attempt to reduce the systemic and local negative effects of chemotherapy, several drugs have been proposed in the literature, the majority of which are immune-modulatory, 
with the purpose of controlling the development of detrimental side effects throughout the course of treatment (12-14).

Pentoxifylline (PTX) was introduced in the 1990s as a prophylactic drug used for the prevention of thrombosis, as it reduces platelet aggregation and the plasma concentrations of fibrinogen, optimizing the perfusion of the microvasculature (15). Platzer et al (16) and Fujimoto et al (17) also investigated the anti-inflammatory effects of PTX and suggested that PTX inhibits the migration and activation of human lymphocytes and is able to modulate the reactions mediated by T lymphocytes. Of note, PTX is well tolerated at high dosages, with no severe side effects (18).

Atorvastatin, a type of statin, synthetically derived from the fermentation of Aspergillus terreus, is a known agent used for the control of hypercholesterolemia (19-21). In addition to affecting the metabolism of cholesterol, its anti-inflammatory properties have been associated with its ability to modulate the levels of C-reactive protein and nitric oxide by upregulating the expression of nitric oxide synthase (21-23).

Trans-caryophyllene, isolated from a resinous oil extracted from the Copaiba tree, has anti-inflammatory and analgesic properties due to its direct vascular action, modulating capillary permeability and blood perfusion (24-27).

The aim of the present study was to evaluate, through a complete leukocyte count, the systemic immunomodulation potential of PTX, atorvastatin and trans-caryophyllene, as well as the possible prophylactic role of these drugs against secondary leukopenia, in an experimental chemotherapy model induced by 5 -FU in Wistar rats.

\section{Materials and methods}

Animals. A total of 32 male Wistar rats were used, with an average age of 7 weeks and average body weight of $220 \mathrm{~g}$. The entire experiment was performed at the Laboratory of Immunopathology and Experimental Pathology, Center of Reproductive Biology, Federal University of Juiz de Fora (MG, Brazil) and was approved by the Animal Research Committee (notion no. 062/2011). The animals were kept in the bioterium in pairs, in cages quilted with shavings, at room temperature and controlled lighting, with ad libitum access to water and feed.

Prophylaxis against leukopenia secondary to experimental chemotherapy with 5-FU. Of the 32 animals used in the experiment, 24 were submitted to treatment with atorvastatin, PTX and trans-caryophyllene prior to the administration of chemotherapy and, subsequently, were randomly divided into the following experimental groups: Group II, animals treated with trans-caryophyllene $(\mathrm{n}=8)$; group III, animals treated with PTX $(n=8)$; and group IV, animals treated with atorvastatin $(n=8)$.

A control group was also established and comprised animals submitted to experimental chemotherapy priorly treated with saline solution alone $(n=8)$ (group I).

Trans-caryophyllene was administered to the animals of group II at a dosage of $50 \mathrm{mg} / \mathrm{kg} /$ day $(24,28)$ through force-feeding, starting 3 days prior to the administration of 5-FU and for 3 days after the last administration of 5-FU, totalling 9 days of treatment.
PTX was administered to the animals of group III over 15 continuous days prior to the administration of chemotherapy by intraperitoneal injection, dissolved in sterile saline solution to a concentration of $150 \mathrm{mg} / \mathrm{ml} /$ day for a total of 18 days of treatment $(4,29)$.

The animals of group IV received $10 \mathrm{mg} / \mathrm{kg} / \mathrm{day}$ of micro-emulsion of atorvastatin by intraperitoneal injection, initiated 1 week prior to the treatment with 5-FU and during the entire course of treatment, totalling 10 days of medication (19).

The control group (group I) received $0.9 \%$ saline solution by peritoneal injections, following the same protocol of the animals on group III.

Experimentalchemotherapytreatment. Theanimals received two intraperitoneal administrations of 5-FU (Eurofarma, São Paulo, $\mathrm{SP}$, Brazil), at the distinct dosages of $100 \mathrm{mg} / \mathrm{kg}$ (1st day) and $60 \mathrm{mg} / \mathrm{kg}$ (2 days after the first application) (30).

Leukocyte count. The animals $(\mathrm{n}=32)$ were euthanized with an overdose of anesthetic $(100 \mathrm{mg} / \mathrm{kg}$ ketamine and $10 \mathrm{mg} / \mathrm{kg}$ xylazine) and their blood was obtained through cardiac puncture. Following collection, the blood was transferred to tubes containing the anticoagulant K2-EDTA.

The global leukocyte count was performed with a Neubauer chamber and the pocH-100iv Automated Hematology Analyzer (Sysmex America, Inc., Lincolnshire, IL, USA) at the Laboratory of Immunopathology and Clinical Immunology (UFJF), at the Center of Reproductive Biology, Federal University of Juiz de Fora, MG, Brazil.

Turk's fluid was used to dilute the blood (standard, 1/20). Following dilution and homogenization, the space between the chamber and the coverslip was carefully filled out to avoid bubble formation.

The leukocyte count was performed under a Bioval binocular microscope, model L2000 A (Bioval, Tocantins, Brazil) at a magnification of $\mathrm{x} 400$ at the lateral sets of four quadrants of the Neubauer chamber and the results were expressed in global quantification of leukocytes per $\mathrm{mm}^{3}$ of blood.

Statistical analysis of the data. The Shapiro-Wilk test was used to verify normality and the Kruskal-Wallis test was used for negative data in the normality test. The analysis was performed using GraphPad Prism $5^{\circledR}$ software (GraphPad Software, Inc., San Diego, CA, USA).

\section{Results}

Leukocyte count normality standard. The normality standard of the leukocyte count for Wistar rats of average age and weight compatible with the sample, raised and maintained at the bioterium used in this research, corresponds to an average of 10,840 leukocytes $/ \mathrm{mm}^{3}$. Of note, the normal value refers to the animal's colony of origin; therefore, not only this global value of the colony must be considered for the analysis of data, but also the reference value of the control group of the experiment (31).

Leukocyte count values. The following values (mean \pm standard deviation) in terms of total leukocyte count were observed

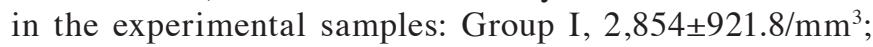




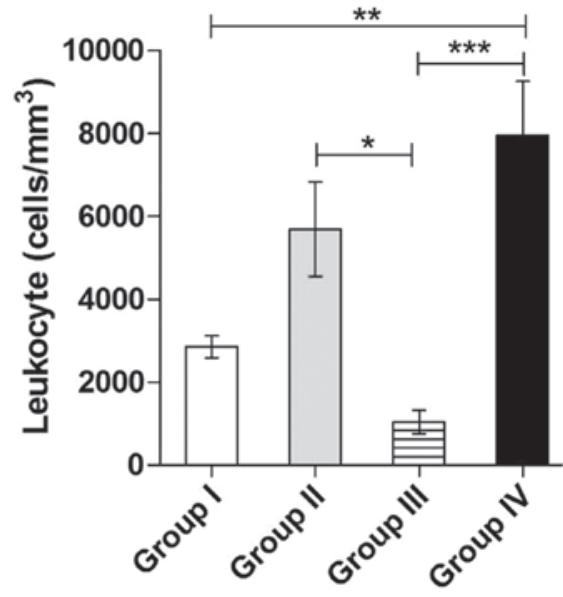

Figure 1. The total leukocyte count of group I (control; animals submitted to experimental chemotherapy and treated prophylactically with saline solution) was $2,854 \pm 921.8 / \mathrm{mm}^{3}$, of group II (animals submitted to experimental chemotherapy and treated prophylactically with trans-caryophyllene), $5,688 \pm 2,284 / \mathrm{mm}^{3}$, of group III (animals submitted to experimental chemotherapy and treated prophylactically with pentoxifylline) $1,042 \pm 694.6 / \mathrm{mm}^{3}$ and of group IV (animals submitted to experimental chemotherapy and treated prophylactically with atorvastatin) $7,944 \pm 3,707 / \mathrm{mm}^{3}$. The results are expressed as mean \pm standard deviation. ${ }^{*} \mathrm{P}<0.05 ;{ }^{* *} \mathrm{P}<0.01 ;{ }^{* * *} \mathrm{P}<0.001$.

group II, 5,688 $22,284 / \mathrm{mm}^{3}$; group III, $1,042 \pm 694.6 / \mathrm{mm}^{3}$; and group IV, $7,944 \pm 3,707 / \mathrm{mm}^{3}$. As regards the normal reference leukocyte count of the colony, the number of leukocytes $/ \mathrm{mm}^{3}$ decreased by $73.67 \%$ (group I, control), $47.52 \%$ (group II, trans-caryophyllene), $90.38 \%$ (group III, PTX) and $26.72 \%$ (group IV, atorvastatin). The results are presented in Fig. 1.

The results demonstrated that the animals of the control group (submitted to experimental chemotherapy and priorly treated with saline solution alone) and of group III (treated prophylactically with PTX) developed more severe leukopenia compared to the other groups; in addition, the group treated with PTX developed more severe leukopenia compared to the control group.

The animals of group II (treated with trans-caryophyllene) did not exhibit a significantly improved leukocyte count compared to that of the control group. However, the animals of group IV (treated prophylactically with atorvastatin) exhibited only a minor decrease in the number of leukocytes, compared to the normal colony parameters (Fig. 1).

\section{Discussion}

PTX has been described in the literature as a TNF- $\alpha$ inhibitor in several pathological conditions, in experimental and clinical studies (32). TNF- $\alpha$ was first described as a protein named cachexin, which was associated with weight loss and other complications observed during the establishment of a cachexia state. In the present study, the animals administered 5-FU and preventively treated with PTX exhibited an exacerbation of the leukopenia.

The secondary infection that developed in this group during the experiment was likely a consequence of the leukopenia associated with PTX administration. The blending episodes of leukopenia and secondary infection explain the deterioration in the overall condition of the rats. In addition, PTX-treated rats presented with blending episodes of epistaxis, secondary skin infections and aggravation of systemic symptoms.

The improvement in leukopenia associated with the preventive use of trans-caryophyllene in group II may be explained by its anti-inflammatory properties, mediated by acting directly on blood vessels, modulating capillary permeability. Trans-caryophyllene has been reported to possess a number of pharmacological properties, including antimicrobial and analgesic properties; additionally, it acts on intestinal smooth muscle, blocking the electromechanical and pharmacomechanical excitation-contraction coupling. Due to these activities, trans-caryophyllene is considered as a potential antispasmodic agent in tracheal smooth muscle (33). Therefore, further studies should be conducted to investigate the novel therapeutic possibilities of this molecule. As an analgesic and anti-inflammatory medication, trans-caryophyllene may improve the quality of life of patients undergoing chemotherapy.

Atorvastatin administration significantly prevented the loss of leukocytes compared to the other groups in this experiment at several relevance levels; therefore, this drug demonstrated the best potential regarding the control of leukopenia in animals submitted to experimental chemotherapy.

Similar results were reported by Medeiros et al (34), who conducted a study in an experimental hamster model with the purpose of investigating the occurrence of ulcerated lesions in the buccal mucosa and its asociation with leukocyte count in groups treated with different dosage and treatment duration. The animals were administered 5-FU (on the first and second days, at dosages of 60 and $40 \mathrm{mg} / \mathrm{kg}$, respectively) and treated with atorvastatin at a dosage of 1,5 and $10 \mathrm{mg} / \mathrm{kg}$, administered $30 \mathrm{~min}$ prior to the daily dose of the 5-FU for 5 or 10 days. The results demonstrated that atorvastatin at a dosage of $5 \mathrm{mg} / \mathrm{kg}$ amplified the leukopenia (34).

Atorvastatin, initially used as a modulator of hypercholesterolemia, has been recently associated with different functions, such as protection against microvascular damage, antifibrotic and anticalcifying effects; in addition, it exhibits antioxidant properties if administered in high doses for the pretreatment of lesions of the myocardium in heart failure, renal disease and abdominal surgery (35-37). These functions are due to the effect of this drug on populations of mononuclear cells, for example the subpopulations of TCD4+ lymphocytes in reciprocity with or independent from the population of cells of the mononuclear system, such as tissue macrophages, Kupffer cells in the liver, microglial cells in the central nervous system and mucosal dendritic cells (38-40). Furthermore, extremely relevant to the chemotherapy model, atorvastatin was suggested by Su et al (38) as a possible anti-apoptotic drug for renal epithelial cells.

Finally, the results of this study suggest that trans-caryophyllene and atorvastatin may be able to prevent leukopenia. Atorvastatin is a drug commonly used for several human pathological conditions. Therefore, this experimental study may provide a basis for further experimental studies and clinical trials investigating the potential of atorvastatin in preventing leukopenia during chemotherapy in cancer patients.

In conclusion, among the selected drugs, atorvastatin exhibited the best preventive potential in regards to leukopenia secondary to experimental chemotherapy with 5-FU, compared 
to the group treated with saline solution, while PTX amplified such alterations in the leukograms of the animals in this trial.

\section{Acknowledgements}

The authors would like to thank Akinory Nagato, for providing guidance with the statistical methodology.

\section{References}

1. McCarthy GM, Awde JD, Ghandi H, Vincent M and Kocha WI: Risk factors associated with mucositis in cancer patients receiving 5-fluorouracil. Oral Oncol 34: 484-490, 1998.

2. Schirmer EM, Ferrari A and Trindade LCT: Oral mucositis evolution after nutritional intervention in cancer patients under palliative care. Rev Dor 13: 141-146, 2012.

3. Sonis ST: New thoughts on the initiation of mucositis. Oral Dis 16: 597-600, 2010.

4. Lima V, Vidal FD, Rocha FA, Brito GA and Ribeiro RA: Effects of tumor necrosis factor-alpha inhibitors pentoxifylline and thalidomide on alveolar bone loss in short-term experimental periodontal disease in rats. J Periodontol 75: 162-168, 2004.

5. Mehta D and Malik AB: Signaling mechanisms regulating endothelial permeability. Physiol Rev 86: 279-367, 2006.

6. Lalla RV, Sonis ST and Peterson DE: Management of oral mucositis in patients who have cancer. Dent Clin North Am 52: 61-77, viii, 2008.

7. Sonis ST: Mucositis as a biological process: a new hypothesis for the development of chemotherapy-induced stomatotoxicity. Oral Oncol 34: 39-43, 1998.

8. Liu W, Zhang CC and Li K: Prognostic value of chemotherapy-induced leukopenia in small-cell lung cancer. Cancer Biol Med 10: 92-98, 2013.

9. Sonis ST: The pathobiology of mucositis. Nat Rev Cancer 4: 277-284, 2004

10. Stringer AM: Interaction between host cells and microbes in chemotherapy-induced mucositis. Nutrients 5: 1488-1499, 2013.

11. Pinedo HM and Peters GF: Fluorouracil: biochemistry and pharmacology. J Clin Oncol 6: 1653-1664, 1988.

12. Alvariño-Martín C, Sarrión-Pérez MG: Prevention and treatment of oral mucositis in patients receiving chemotherapy. J Clin Exp Dent 6: e74-e80, 2014.

13. Owlia F, Kazemeini SK and Gholami N: Prevention and management of mucositis in patients with cancer: a review article. Iranian J Cancer Prevent 5: 216-220, 2012.

14. Sonis S and Clark J: Prevention and management of oral mucositis induced by antineoplastic therapy. Oncology (Williston Park) 5: 11-18; discussion 18-22, 1991.

15. Sampaio EP, Moraes MO, Nery JA, Santos AR, Matos HC and Sarno EN: Pentoxifylline decreases in vivo and in vitro tumour necrosis factor-alpha (TNF-alpha) production in lepromatous leprosy patients with erythema nodosum leprosum (ENL). Clin Exp Immunol 111: 300-308, 1998.

16. Platzer C, Meisel C, Vogt K, Platzer M and Volk HD: Up-regulation of monocytic IL-10 by tumor necrosis factor-alpha and cAMP elevating drugs. Int Immunol 7: 517-523, 1995.

17. Fujimoto T, Nakamura T, Furuya T, et al: Relationship between the clinical efficacy of pentoxifylline treatment and elevation of serum $\mathrm{T}$ helper type 2 cytokine levels in patients with human T-lymphotropic virus type I-associated myelopathy. Intern Med 38: 717-721, 1999.

18. González-Amaro R, Portales-Pérez D, Baranda L, et al: Pentoxifylline inhibits adhesion and activation of human T lymphocytes. J Immunol 161: 65-72, 1998.

19. Azevedo ÍM, Kumakura HS, Alloufa SL, et al: Effect of simvastatin in attenuation of mucositis induced by methotrexate in rats. J Surg Clin Res 1: 22-32, 2010.

20. Lea AP and McTavish D: Atorvastatin. A review of its pharmacology and therapeutic potential in the management of hyperlipidaemias. Drugs 53: 828-847, 1997.

21. Veillard NR and Mach F: Statins: the new aspirin? Cell Mol Life Sci 59: 1771-1786, 2002.

22. Ponomarev ED, Maresz K, Tan Y and Dittel BN: CNS-derived interleukin-4 is essential for the regulation of autoimmune inflammation and induces a state of alternative activation in microglial cells. J Neurosci 27: 10714-10721, 2007.
23. Weis M, Heeschen C, Glassford AJ and Cooke JP: Statins have biphasic effects on angiogenesis. Circulation 105: 739-745, 2002.

24. Dias DS, Fontes LB, Crotti AE, et al: Copaiba oil suppresses inflammatory cytokines in splenocytes of $\mathrm{C} 57 \mathrm{Bl} / 6$ mice induced with experimental autoimmune encephalomyelitis (EAE). Molecules 19: 12814-12826, 2014.

25. Leandro LM, Vargas Fde S, Barbosa PC, Neves JK, da Silva JA and da Veiga-Junior VF: Chemistry and biological activities of terpenoids from copaiba (Copaifera spp.) oleoresins. Molecules 17: 3866-3889, 2012.

26. Vilanova C, Ribeiro SM, Machado R, et al: Evaluation of oil-resin activity of Copaifera sp. on gastric emptying in Rattus novergicus. Emirates J Food Agriculture 25: 394-397, 2013.

27. Dias DS, Fontes LBA, Crotti AEM, et al: Copaiba Oil Suppresses Inflammatory Cytokines in Splenocytes of C57B1/6 Mice Induced with Experimental Autoimmune Encephalomyelitis (EAE). Molecules 19:12814-12826, 2014

28. Fernandes ES, Passos GF, Medeiros R, et al: Anti-inflammatory effects of compounds alpha-humulene and (-)-trans-caryophyllene isolated from the essential oil of Cordia verbenacea. Eur J Pharmacol 569: 228-236, 2007.

29. Zargari O: Pentoxifylline: a drug with wide spectrum applications in dermatology. Dermatol Online J 14: 2, 2008.

30. Sonis ST, Tracey C, Shklar G, Jenson J and Florine D: An animal model for mucositis induced by cancer chemotherapy. Oral Surg Oral Med Oral Pathol 69: 437-443, 1990.

31. Lillie LE, Temple NJ and Florence LZ: Reference values for young normal Sprague-Dawley rats: weight gain, hematology and clinical chemistry. Hum Exp Toxicol 15: 612-616, 1996.

32. Corrêa JO, Aarestrup BJ and Aarestrup FM: Effect of thalidomide and pentoxifylline on experimental autoimmune encephalomyelitis (EAE). Exp Neurol 226: 15-23, 2010.

33. Pinho-da-Silva L, Mendes-Maia PV, Teófilo TM, et al: trans-Caryophyllene, a natural sesquiterpene, causes tracheal smooth muscle relaxation through blockade of voltage-dependent $\mathrm{Ca}^{2+}$ channels. Molecules 17: 11965-11977, 2012.

34. Medeiros CA, Leitao RF, Macedo RN, et al: Effect of atorvastatin on 5-fluorouracil-induced experimental oral mucositis. Cancer Chemother Pharmacol 67: 1085-1100, 2011.

35. He GX and Tan W: High-dose atorvastatin pretreatment could diminish microvascular impairment in patients undergoing elective percutaneous coronary intervention. J Geriatr Cardiol 10: 355-360, 2013.

36. Kose E, An T, Kikkawa A, Matsumoto Y and Hayashi H: Effects on serum uric acid by difference of the renal protective effects with atorvastatin and rosuvastatin in chronic kidney disease patients. Biol Pharm Bull 37: 226-231, 2014.

37. Liu D, Cui W, Liu B, et al: Atorvastatin protects vascular smooth muscle cells from TGF- $\beta 1$-stimulated calcification by inducing autophagy via suppression of the $\beta$-catenin pathway. Cell Physiol Biochem 33: 129-141, 2014.

38. Su Q, Li L, Liu Y, Zhou Y, Wang J and Sun Y: Effect of intensive atorvastatin therapy on periprocedural PDCD4 expression in $\mathrm{CD}^{+} \mathrm{T}$ lymphocytes of patients with unstable angina undergoing percutaneous coronary intervention. Cardiology 127: 169-175, 2014.

39. Wang W, Song W, Wang Y, Chen L and Yan X: HMG-CoA reductase inhibitors, simvastatin and atorvastatin, downregulate ABCG1-mediated cholesterol efflux in human macrophages. J Cardiovasc Pharmacol 62: 90-98, 2013.

40. Weber MS, Prod'homme T, Youssef S, Dunn SE, Steinman L and Zamvil SS: Neither T-helper type 2 nor Foxp $3^{+}$regulatory T cells are necessary for therapeutic benefit of atorvastatin in treatment of central nervous system autoimmunity. J Neuroinflammation 11: 29, 2014. 